\title{
Modification in the Retropharyngeal Flap for Velopharyngeal Insufficiency with a Refractory Palate Fistula: Case Report
}

\section{Araujo-López Adan ${ }^{1, *}$, Barragán-Chávez Jean Carlo², González-Rodríguez Mónica1 ${ }^{1}$ Pierdant-Lozano Rene ${ }^{3}$, Azcarate-Varela José Andrés ${ }^{3}$, and Caracheo-Rodríguez Raul Cervando ${ }^{3}$}

${ }^{1}$ General Surgeon, Ambulatory Unit of Specialties in Ciudad Valles, San Luis Potosí, General Hospital of Mexico, Plastic and Reconstructive Service, General Surgeon Service, Mexico

2Plastic Surgeon Resident, Ambulatory Unit of Specialties in Ciudad Valles, San Luis Potosí, General Hospital of Mexico, Plastic and Reconstructive Service, General Surgeon Service, Mexico

${ }^{3}$ Plastic Surgeon, Ambulatory Unit of Specialties in Ciudad Valles, San Luis Potosí, General Hospital of Mexico, Plastic and Reconstructive Service, General Surgeon Service, Mexico

*Corresponding author: Araujo-López Adan, General Surgeon, Ambulatory Unit of Specialties in Ciudad Valles, San Luis Potosí, General Hospital of Mexico, Plastic and Reconstructive Service, General Surgeon Service, Mexico, Tel: +52 (444) 4 49 12 03; E-mail: draraujolopez@gmail.com

Received: 11 Oct, 2019 | Accepted: 01 Nov, 2019 | Published: 06 Nov, 2019

Citation: Araujo-López A, Barragán-Chávez JC, González-Rodríguez M, Pierdant-Lozano R, Azcarate-Varela JA, et al. (2019) Modification in the Retropharyngeal Flap for Velopharyngeal Insufficiency with a Refractory Palate Fistula: Case Report. J Surg Open Access 5(5): dx.doi. org/10.16966/2470-0991.198

Copyright: (c) 2019 Araujo-López A, et al. This is an open-access article distributed under the terms of the Creative Commons Attribution License, which permits unrestricted use, distribution, and reproduction in any medium, provided the original author and source are credited.

\section{Abstract}

Introduction: Velopharyngeal Dysfunction (VPD) refers to any situation in which an individual is unable to completely close the nasal airway during speech.

Case report: Male of 16 years, lip and cleft palate post operated, with a velopharyngeal dysfunction and a fistula. We covered by an incision closing the middle line, with a satisfactory evolution.

Discussion: The goal of surgical intervention is to produce or restore velopharyngeal competence while avoiding the complications of upper airway obstruction.

Conclusion: Dysfunction velopharyngeal is the most frequent cause of hyper nasal speech, and most of them have anterior palate fistula, the posterior retropharyngeal flap is one of the one of the best post-surgical evolution.

Keywords: Left lip and palate; Posterior pharyngeal flap; Velopharyngeal insufficiency

\section{Introduction}

Normal speech is dependent upon the functional and structural integrity of the velopharynx, a complex and dynamic structure that serves to uncouple the oral and nasal cavities during sound production [1]

Velopharyngeal dysfunction (VPD) refers to any situation in which an individual is unable to completely close the nasal airway during speech. The velopharyngeal mechanism is comprised of a complex group of structures that act in unison to control airflow through the nose and mouth by elevation the soft palate and constriction of both the lateral and posterior pharyngeal walls [2].

Velopharyngeal dysfunction simple denotes that it will have an incomplete closure, the cause is found in cleft palate, submucous palatal fistulae, post adenoidectomy, and neuromuscular disorders or persists after cleft palate surgery or removal of tumors, instead of that Rodriguez E, et al. [1] says that in some patients is so hard to get the cause, saying that there won't have an etiology.
It is seen roughly 20 to $30 \%$ of individuals who have undergone cleft palate repair and 5 to $10 \%$ of patients with a submucous cleft palate $[1,2]$.

Any disruption in this mechanism may result in abnormal, poorly intelligible speech, having a hypernasality, nasal emission, decrease vocal intensity, and/or facial grimacing [3].

Using a posterior pharyngeal flap or the creating of midline flaps from the posterior pharyngeal wall represents the oldest surgical technique for the management of the VPD. The pharyngeal flap functions primarily as a central obturator of the velopharyngeal port [1-4]. Since its original description by Schoenborn and popularization by Gosain AK, et al. [5] posterior pharyngeal flap has been the most widely and effectively used procedure for Velopharyngeal Insufficient.

\section{Case Report}

Male of 16 years, with a right complete palate and lip cleft, at the age of 5 months he gets cheiloplasty, and at 18 months the palatoplasty. 
The patient did not had a stable economy, having lack of complete speech therapy (by using phonemes $<$ CHI-LLA $>,\langle\mathrm{I}\rangle$, $<\mathrm{U}>$, e $<$ I $>$ long pronunciation), his breathe was hard but not with a dyspnea problem, having a torpid evolution by the closure of the fistula and the persistence of the nasal speechless even with an oral therapy. We evaluated during a rural campaign of clef and lip palate in San Luis Potosí, after a nasopharyngoscopy that confirm a sagittal velopharyngeal dysfunction with good lateral wall motion and Fistula tip IV of the Pittsburg Classification System (Hard palate fistula of $14 \mathrm{~mm}$ ) (Figure 1), we decided to program for an elective posterior retropharyngeal flap (Figure 2) during surgery we decide to cover the anterior palate fistula with the posterior retropharyngeal flap (by a 2 hours surgery, and 1 day stay at hospital), we implement a modification in the technique (Figure 3) by opening the middle line and have the improvement to connect to the fistula so we covered to retropharyngeal space, extending and suturing to the floor of nasal space until the fistula full cover, and close the palate, having a successfully post-surgical without problem of breathing, he had vocal therapy, until six months in the new campaign the fistula close and the nasal speechless disappear, completing phrases with phonemes "I", "U" and long "I" pronunciation (Figure 4).

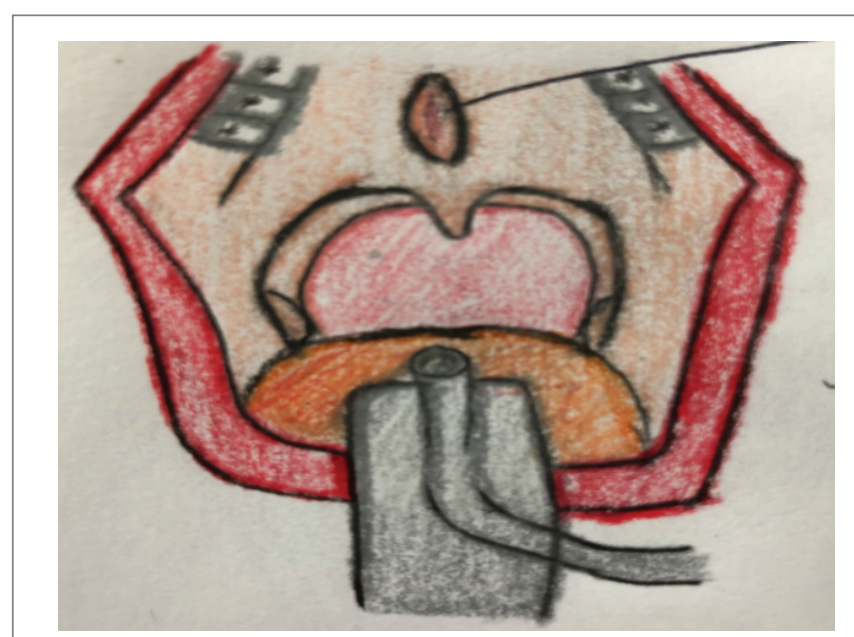

Figure 1: Fistula type IV of hard palate with sagittal velopharyngeal dysfunction and good wall.

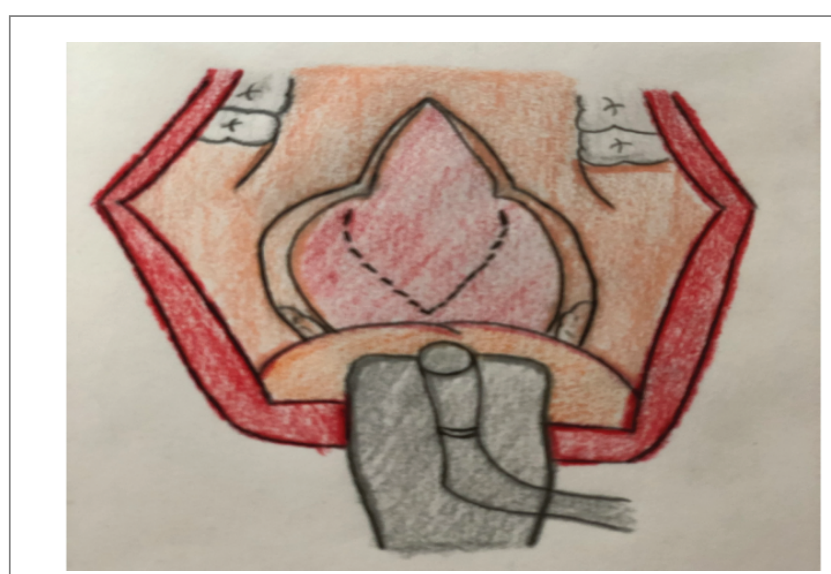

Figure 2: Posterior pharyngeal flap in triangle to close the middle line with the fistula type IV of Pittsburg.

\section{Discussion}

The velopharyngeal sphincter is situated between the oral and nasal cavities and permits the speaker to separate the nasal cavity from the oral cavity. Velopharyngeal closure is achieved by tension of the velum and its elevation toward the pharyngeal walls that move toward the rising velum and diminish the lumen of the velopharynx [1]. The velopharyngeal port is defined anteriorly by the soft palate, or velum, laterally by the lateral pharyngeal walls, and posteriorly by the posterior pharyngeal wall [2].

Anatomic causes are most common and are typically associated with a previously repaired cleft palate associated in 20 to $30 \%$, Gosain $\mathrm{AK}$, et al. [5] refers more than these, but we are in the average [2,5].

Fistulas anywhere within the palate can lead to abnormal intraoral air escape, and tonsillar hypertrophy or scarring of the posterior tonsillar pillars can also serve as a barrier to normal closure of the velum against the posterior pharyngeal wall $[1,2]$.

Although palatal fistula is common after cleft palate repair, up to $34 \%$, minimal literature regard its management available [2,3].

Velopharyngeal insufficiency is found in cleft palate, submucous palatal fistulae, post adenoidectomy, and neuromuscular disorders or persists after cleft palate surgery or removal of tumors [3].

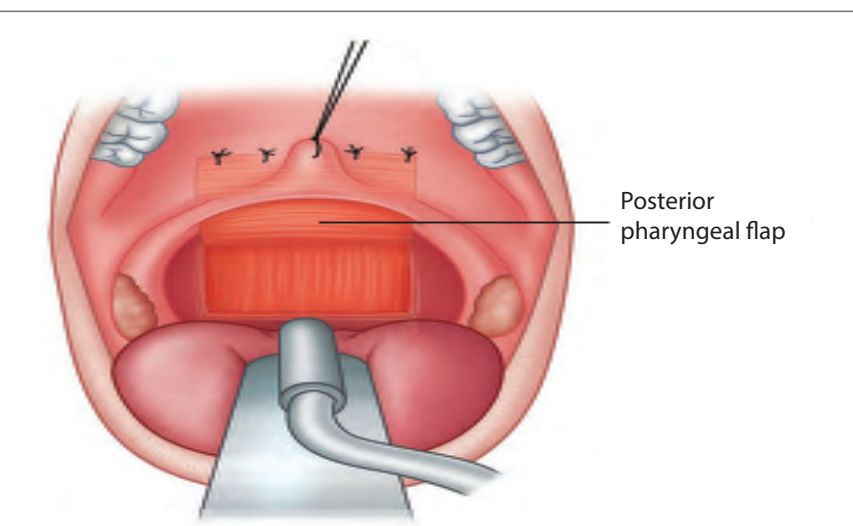

Figure 3: Posterior pharyngeal flap (muscular-mucosa flap) conventional surgery.

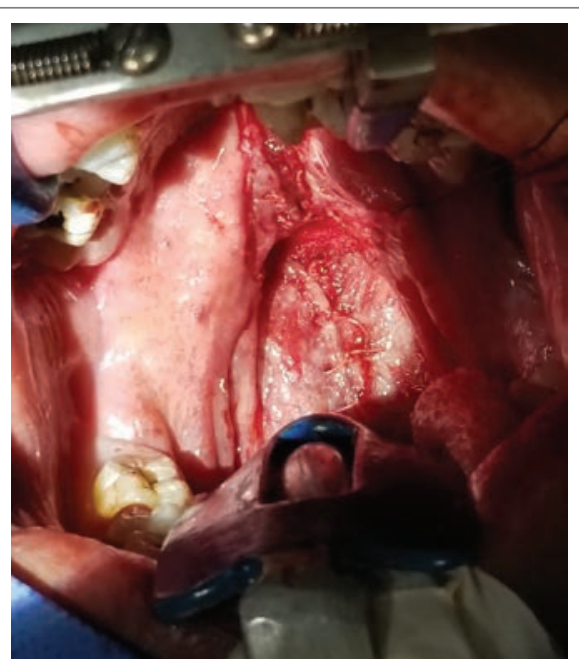

Figure 4: Posterior pharyngeal flap, with a triangle modification, closing full nasopharyngeal flap. 


\section{Non-surgical treatment options}

The options are having a prosthetic but are just temporal or just like a permanent solution. Prostheses typically are available in the form of a palatal lift or an obturator. Soft palate obturator or speech aid prostheses are more effective in velopharyngeal insufficiency, where the palate has inadequate tissue length $[2,4]$.

\section{Surgical treatment options}

There are numerous surgical treatments VPD including posterior pharyngeal wall augmentation, sphincter pharyngoplasty, palatal lengthening procedures (Furlow palatoplasty and pushback palatoplasty), and posterior pharyngeal flaps.

The common aim of these procedures is to create a permanent partial obstruction of the velopharyngeal space in order to correct hyper nasal speech $[3,4]$.

Pharyngeal flap is created by suturing an inferiorly based or more commonly a superiorly based posterior pharyngeal wall flap to the soft palate to bridge the central portion of the velopharyngeal gap, leaving a lateral port on each side for breathing [3]. The goal of this procedure is to recruit tissue from the posterior pharyngeal wall and attach it to the soft palate to bridge the central portion of the large velopharyngeal gap [2-4]. Several modifications have been added to limit complications and improve results [5]

The width of the pharyngeal flap should be tailored to the functional and anatomic needs of each patient, again as determined by preoperative imaging $[1,5]$.

The pharyngeal flap opening the middle line, until the fistula to get close, doing a pharyngeal flap with the mucosa and pharyngeal muscle, this flap is in triangle because is the best option to close the fistula, making sure lateral space with a nasal tube (Figure 3) [Posterior pharyngeal flap conventional technique].

\section{Complications}

Complications of pharyngeal flap surgery include bleeding, dehiscence, and nasal airway obstruction, including obstructive sleep apnea $[3,5]$.
Off all complications obstructive sleep apnea is the most serious of the pharyngeal flap surgery. It was estimated to occur in up to $20 \%$ of patients $[3,5]$.

The vast majority of patients demonstrate resolution of clinical and polysomnographic evidence of nocturnal upper airway obstruction within several months of surgery, as edema subsides [5].

\section{Conclusion}

The L-shaped posterior pharyngeal flap is one of the most common improved in VPD, but a modification in it (doing a triangle flap, opening the middle line), will close with more efficient an anterior palate fistula, having less complications, than others technique compare conventional, and a full close of the fistula in the future.

\section{Financing}

For the realization of this article. Confirm that this information has been included in the financing section of the manuscript.

\section{Conflict of Interests}

We declare that we have no conflicts of interest.

\section{References}

1. Rodriguez ED, Neligan PC, Losee JE (2012) Plastic Surgery: Craniofacial, Head and Neck Surgery and Pediatric Plastic Surgery. $3^{\text {rd }}$ edition, Elsevier Health Sciences 6: 614-635.

2. Woo AS (2012) Velopharyngeal Dysfunction. Semin Plast Surg 26: 170-177.

3. Elsheikh E, El-Anwar MW (2016) Posterior Pharyngeal Flap for Velopharyngeal Insufficiency Patients: A New L-Shaped Flap. J Craniofac Surg 27: 204-208.

4. El-Anwar MW, Elsheikh E, Askar S (2018) Single-Stage Repair of Palatal Fistula and Velopharyngeal Incompetence by the New L Flap. J Craniofac Surg 29: e70-e73.

5. Gosain AK, Arneja JS (2007) Management of the black hole in velopharyngeal incompetence: combined use of a Furlow palatoplasty and sphincter pharyngoplasty. Plast Reconstr Surg 119: 1538-1545. 